\title{
A real-time Road Boundary Detection Algorithm Based on Driverless Cars
}

\author{
Xuekui ZHU ${ }^{1, a}$, Meijuan $\mathrm{GAO}^{2, \mathrm{~b}}$, Shangnian $\mathrm{LI}^{3, \mathrm{c}}$ \\ ${ }^{1}$ Beijing Union University, Beijing Key Laboratory of Information Service Engineering, Beijing, \\ 100101, China \\ ${ }^{2}$ Beijing Union University, Beijing Key Laboratory of Information Service Engineering, Beijing, \\ 100101, China \\ ${ }^{3}$ Beijing Union University, Beijing Key Laboratory of Information Service Engineering, Beijing, \\ 100101, China
}

aemail: zhuxuekui3@163.com, bemail: gaomeijuan@163.com, ${ }^{\mathrm{c} e m a i l}:$ lishangnian@163.com

Keywords: driverless cars; LIDAR; RANSAC; KALMAN filtering; Road boundary detection

\begin{abstract}
In this study, aiming at road boundary detection problem of driverless cars in structured and semi-structured roads, the paper proposed a new real-time road boundary detection algorithm based on driverless cars as a platform. The algorithm firstly included following steps: calibration, layered and median filtering according to the obtained LIDAR point cloud data, and then extract the left and right road boundary points of each layer. The extracted road boundary points are then straight line fitted using RANSAC algorithm. Finally, straight line is tracked using KALMAN filtering, then achieve real-time road boundary detection. The test results show that this boundary detection algorithm has the high accuracy and reliability and is able to accurately accomplish the boundary detection task, which can satisfy the requirements of real-time system. And this algorithm has been applied successfully in "The future challenge competition for driverless cars" in 2014 year and has obtained the third place.
\end{abstract}

\section{Introduction}

Since the birth of intelligent robots, it has become more and more popular on the study of driverless cars. Among them, the road boundary detection is a important research content, attracting the attention of many scholars. The road boundary detection relies on driverless cars mounted sensors to perceive the surrounding environment information. To detect road boundaries, many methods using different types of sensors, including cameras, radar and LIDAR sensors, have been studied. Some of them, LIDAR sensors can give high accuracy and resolution range data with a wide FOV. What is more, their capability in adverse weather conditions has also been improving. Due to these, LIDAR sensors have been widely used.

There are many different methods about road boundary detection proposed. Detected data points is straight line fitted, and fuse information of four scan lines to get fused road-boundary(Yang et al, 2013) [1]. And then according to motion information of the vehicle, constructing KALMAN filter is to track and estimate the road-boundary and intersection. Using a downward-looking LIDAR sensor estimates the roll and pitch angles of the sensor relative to the scanning road surfaces by using an IPDAF (Han et al, 2012) [2]. These estimated roll and pitch angles are applied to classify the extracted line segments into road and obstacle line segments when the probability of the track existence, which is given by the IPDAF, is larger than the threshold value [3]. It achieves good results but need a large amount of calculation. Chen calculates fuzzy cluster centers directly and predicts the states and predictable error simultaneously, and calculates membership values of current state based on the maximum entropy principle (Chen et al. 2007) to detect road boundary [4]. A scholar uses a LIDAR and extended KALMAN filter algorithm to detect road boundary, and to track [5]. From the above methods, we can find all of them use the motion information of the vehicle and they did not give a solution without the motion information 
of the vehicle.

In addition to a LIDAR, there are four LIDAR, eight LIDAR, thirty second and sixty-four LIDAR. Compared to a LIDAR, multi-line LIDAR has a wider range of point cloud data, and supply more feature points, which makes obtaining stable road boundary as possible. Because the sixty-four LIDAR are expensive, we choose LUX8L sensor as the perception of the surrounding environment. It consists of eight the interval $0.8^{\circ}$ laser scanning constitute a total of $6.4^{\circ}$. This algorithm has been used in "The future challenge competition for driverless cars" in 2014 year and has obtained the third place. The driverless car is shown in Figure 1.

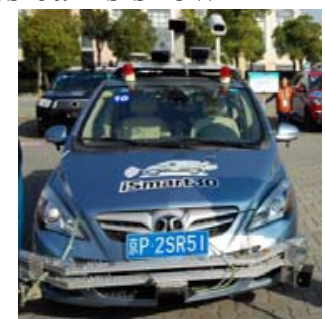

Fig.1.Beijing union university ISmart30 smart car

\section{Principle of edge detection}

Edge detection is a word which first appears in image analysis and processing and it is the key step in the image analysis and processing. It is important for feature description 、 image matching and identification[6].Edge detection mainly recognize salient region in the image and the obvious points. It is important in the image processing and computer vision, especially in the feature extraction .However, the image based on vision sensors is hard for edge detection because of mixing, fuzzy and deformation [7]. The edge detection method is mainly divided into two categories.

1) The classical edge detection algorithm, such as SOBEL operator method, the optimal operator method, etc.

2) The algorithms of image edge detection based on high and new technology, such as edge detection algorithm based on neural network, the edge detection algorithm based on mathematical morphology, etc. This paper mainly studies the road edge detection based on laser radar, which data is a series of points. It is different from the edge detection method based on image.

In this paper, the main steps of road boundary detection algorithm are as follows:

(1) Obtaining eight LASAR data and calibration;

(2) Data classification;

(3) Extraction of each layer of boundary point;

(4) Using RANSAC algorithm to fit line;

(5) Using KALMAN filter to get new road edge.

The program flow chart is shown in figure 2.

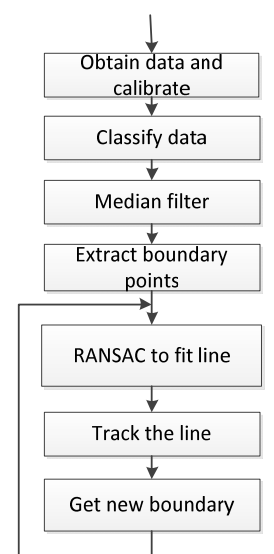

Fig.2. Road boundary detection algorithm program flow chart 


\section{Road boundary Detection}

Firstly, this paper uses API functions provided by the company IBEO to collect data .Due to the height of the radar installation, pitching Angle, slope Angle and rotation Angle, we need calibration data. According to the following coordinate transformation formula, we know:

Rigid body transformation can be made of translational matrix $\mathrm{T}$ and the matrix $\mathrm{R}$ to describe. The conversion formula is as follows [8]:

$$
\left[\begin{array}{c}
X_{V}^{P} \\
Y_{V}^{P} \\
Z_{V}^{P}
\end{array}\right]=R_{3 X 3} *\left[\begin{array}{c}
X_{L}^{P} \\
Y_{L}^{P} \\
Z_{L}^{P}
\end{array}\right]+T_{3 \times 1}
$$

Among them, the $\mathrm{V}$ is coordinate system, the $\mathrm{L}$ is radar coordinate system. The rotation matrix and translation matrix can be merged, as follows:

$$
\left[\begin{array}{c}
X_{V}^{P} \\
Y_{V}^{P} \\
Z_{V}^{P}
\end{array}\right]=\left[\begin{array}{l}
T_{X Y_{2 \times 4}} \\
T_{1 \times 4}
\end{array}\right] *\left[\begin{array}{l}
X_{L}^{P} \\
Y_{L}^{P} \\
Z_{L}^{P} \\
1
\end{array}\right]
$$

In the process of calibration, set on thin rods around the body, record its place in the body coordinate system, and a frame laser radar data, find the location of the thin rods, write down the 3 $\mathrm{d}$ coordinate; Then the car body position unchanged, in car body placed around cardboard boxes, record its in the height of the car body coordinates, and write down the coordinates in the middle of the radar data. To repeat the above steps several times, record the coordinates, then solving the above equation to calculate the corresponding calibration matrix.

Secondly, we use median filtering according to the obtained LIDAR point cloud data, and then extract the left and right road boundary points of each layer. According to the data distribution characteristics of road model, we need to filter the raw data in the longitudinal axis, making the data smoother. The results are shown in Figure 3(a) and (b).

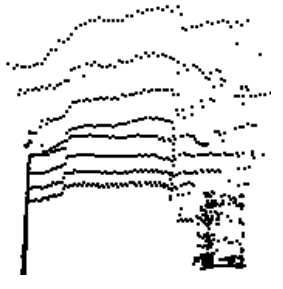

(a)before median filtering

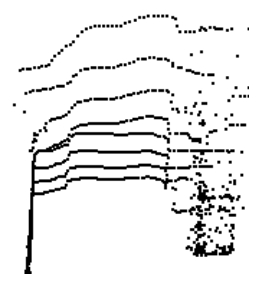

(b) after median filtering

Fig.3.Median filtering

Thirdly, at a beginning of center of the vehicle, we seek the both sides and divide the raw data into left and right boundary point. In order to solve the obstacles in the middle of the road, we cannot look for a boundary point, we must look for several points per layer. According to the height of the boundary of the road, we can set the arbitrary threshold to improve the accuracy of detection [10]. Point to one layer, we need to select eligible points from the points. The screening procedure is as follows: first of all, we choose left and right point from the left and right boundary points and then generate a straight line. According to the road width and the range of slope, we can gain a suitable line. The conditions we choose are shown in Figure 4 and formula is as follows:

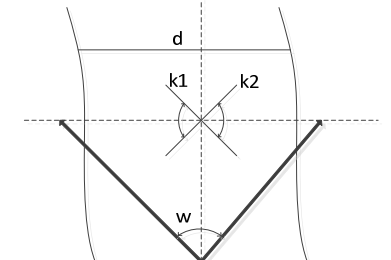

Fig.4.Select the boundary points 


$$
\left\{\begin{aligned}
k_{1} & \leq k_{i} \leq k_{2} \\
\frac{\pi-\omega}{2} & \leq \theta_{i} \leq \frac{\pi+\omega}{2} \\
0 & \leq d_{i} \leq d
\end{aligned}\right.
$$

Including these, $\mathrm{k}$ represents slope, $\omega$ represents view Angle, $\mathrm{d}$ represents road width.

Fourthly, as for linear fitting, the least square is the simplest method. But it is unable to obtain the ideal results because of its poor noise resistance. We use RANSAC algorithm instead of the least square to fit line. In the first step, two points are randomly selected from the left and right road boundary points. A line model and the corresponding model parameters are computed using only the elements of the boundary points. In the second step, the algorithm checks which elements of the entire boundary points are consistent with the line model instantiated by the estimated model parameters obtained from the first step. A data element will be considered as an outlier if it does not fit the fitting model instantiated by the line model parameters within some error threshold that defines the maximum deviation attribute to the effect of noise. The set of inliers obtained for the line model is called consensus set. The RANSAC algorithm will iteratively repeat the above two steps until the obtained consensus set in certain iteration has enough inliers. The results are shown Figure 5.

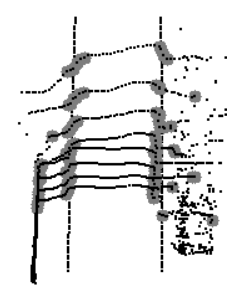

Fig.5.RANSAC algorithm

Lastly, Using the above method can construct left and right road boundary, then using KALMAN filtering to track road boundary. After KALMAN filter, we get a stable road boundary.

\section{Test results}

The road boundary detection algorithm is proposed in this paper is to apply in the environment perception system of driverless cars. The experiment based on driverless cars as a platform mounted 8 LIDAR. Sensors installation is shown in Figure 6.

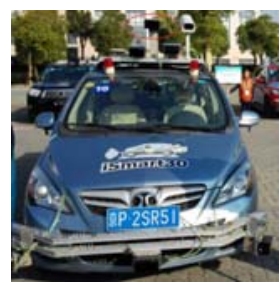

Fig.6.ISmart30 top eight LIDAR installation schematic diagram

Experiment environment 1 is under the viaduct, road width is 10 meters approximately and the road is flat. When we collect raw data, the vehicle is mounted LIDAR that have 2.15 meters height, 3.1 degree pitch angle and 0 degree yaw angle. Under the viaduct, the GPS is no signal. We cannot rely on GPS to move on while the camera cannot provide accurate lane information due to the sunshine. So we must use LIDAR to detect road boundary. The result is shown in Figure 7(a), 7(b) and $7(\mathrm{c})$.

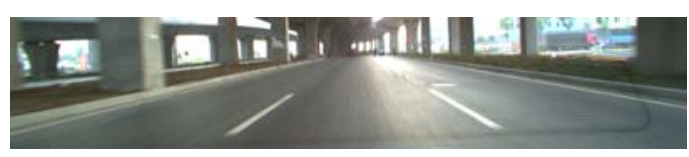

(a)The road under the viaduct
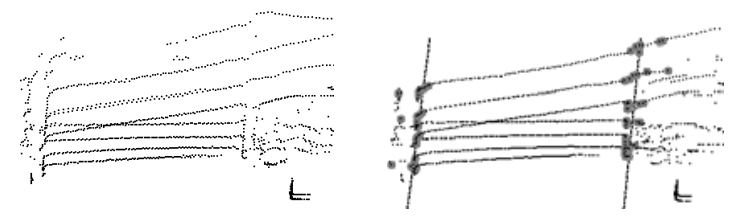

(c) Detected road boundary

Fig.7. The experimental results 1 
Experiment environment 2 is in the campus, road width is 4 meters approximately and the road is uneven. When we collect raw data, the vehicle is mounted LIDAR that have 2.05 meters height , 2.2 degree pitch angle and 0 degree yaw angle. The result is shown in Figure 8(a), 8(b) and 8(c).

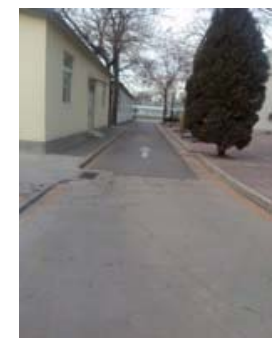

(a)The road in the campus

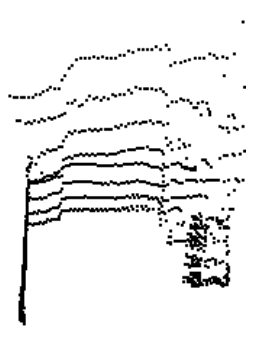

(b) Raw data

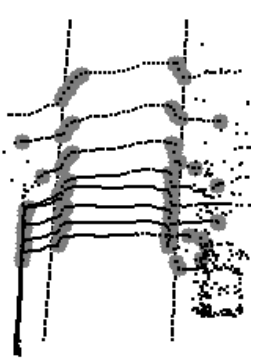

(c) Detected road boundary

Fig.8. The experimental results 2

Judging an algorithm is mainly from three aspects: accuracy, robustness and real-time. The following is mainly from the three aspects of road boundary detection for data analysis. The data that used in this paper are mainly from the raw data, which is collected in different sections and different time by driverless car mounted sensors. Among them, it comes mainly from the Changshu section, suburban section and the Beijing Union University section.

From the data analysis of diverse sections of the road, we can arrive at a conclusion that it will have a certain impact on the algorithm in different sections, obstacles and the motion of vehicles.

From a large number of field tests, we know that the accuracy of the algorithm in structured road is better than that on the unstructured road. In the straight and no obstructions roads, the vehicle running parallel to road boundary approximately. On the highway, the rate of the algorithm general accuracy is up to $90 \%$; while in the rugged road, the rate of accuracy is only $70 \%$.

For the same section of the structured and no obstructions roads, the vehicle running parallel to road boundary approximately, in the absence of obstacles, the rate of the algorithm general accuracy is up to $90 \%$; while in the case of obstruction, the accuracy rate is $70 \%$.

From the analysis, we can know that the road boundary detection is more accurate in this paper and meets the requirement of the driverless cars mounted sensors.

Experiments are implemented in the Intel i7 processor, and the processing time of per frame is $16 \mathrm{~ms}$, which is far less than the LIDAR sampling period of $80 \mathrm{~ms}$. While the general driverless cars decision-making cycle is $100 \mathrm{~ms}$, the algorithm can meet the real-time requirements.

\section{Conclusion}

In this study, a new real-time road boundary detection algorithm based on driverless cars for structured and semi-structured roads was discussed. The accuracy, robustness and timeliness of this algorithm meet the requirements of environment awareness. It is a simple and easy method. It has some advantages. On the one hand, it gives a solution of road boundary without vehicle movement information. On the other hand, it is a real-time way.

From the discussion, we can arrive at a conclusion that it has many further improvement of the algorithm. One of them, the algorithm lacks vehicle information, such as the speed of vehicle, heading of vehicle and so on.

\section{Acknowledgement}

In this paper, the research was sponsored by the project of Construction of Innovative Teams and Teacher Career Development for Universities and Colleges under Beijing Municipality (CIT\&TCD20130513). 


\section{References}

[1]Yang Xiang-jun and so on. Detection and tracking of campus road based on four-line laser radar [J]. Transducer and Microsystem Technologies,2013,32(9):134-138.

[2] Han J Y , et al. Enhanced road boundary and obstacle detection using a downward-looking lidar sensor[J]. IEEE Transactions on Vehicular Technology,2012,61 (3): 971 - 985.

[3]Musicki D,Evans R,Stankovic S.Integrated probabilistic data association [J].IEEE Transactions on Automatic Control,1994,39(6):1237-1241.

[4]Chen De-bao and so on. Quick Road-boundary Detection Based on 2D Laser Rangefinder. Journal of Image and Graphics, 2007,12(9):1604-1609.

[5] Wijesoma W S,Kodagoda K R S,Balasuriya Arjuna P.Roadboundary detection and tracking using ladar sensing[J].IEEE Transactions on Robotics and Automation,2004,20 (3):456 - 464.

[6]Dong Hong-yan. Some technology about edge detection [D].changsha: National university of defense technology,2008.

[7]Jia Lei and so on. The survey of the technology of image edge detection. Technology Wind,2010(4):226.

[8] Ying Tong-tong. Research on 3D LIDAR-based Environment Perception for ALV [D]. changsha: National university of defense technology,2011.

[9]Liu Zi and so on. Real-time Road-boundary Detection Based on 3D Laser [J]. Journal of huazhong university of science and technology, 2011,11:351-354.

[10] Wan zhong - tao. Research on LADAR Based Road and Obstacles [D]. changsha: National university of defense technology,2010.

[11] Chin W. Partial Least Squares Is To LISREL As Principal Components Analysis Is To Common Factor Analysis[J]. Technology Studies, 1995, 2(2):315-319.

[12]Fischler M A, Bolles R C. Random sample consensus: A paradigm for model fitting with applications to image analysis and automated cartography[J].Communication of the ACM,1981(24)381-395.

[13] Choi H C, Park J M,et al. Vision-based fusion of robust lane tracking and forward vehicle detection in a real driving environment[J].International Journal of Automotive Technology,2012,20 (3) : 653-669. 